\title{
Baseline Characteristics and Changes in Bone Mineral Density T-Scores of Bulgarian Women with Postmenopausal Osteoporosis Receiving Denosumab in Routine Clinical Practice
}

\author{
Mihail Boyanov $^{1} \cdot$ Alexander Shinkov $^{2}$ Emi Psachoulia ${ }^{3} \cdot$ Michele Intorcia $^{3} \cdot$ \\ Reneta Petkova $^{4}$
}

Published online: 17 December 2016

(C) The Author(s) 2016. This article is published with open access at Springerlink.com

\begin{abstract}
Background Postmenopausal osteoporosis (PMO) is common among women over 50 years of age and is associated with an increased risk of fracture. Bone-targeted agents, such as denosumab, can reduce fracture risk in patients with PMO.

Objective The aim was to describe baseline characteristics and changes in bone mineral density (BMD) T-scores among women with PMO receiving denosumab in Bulgaria.

Methods This multicenter chart review included women with PMO receiving denosumab for $\geq 1$ year in Bulgaria (October 2011-August 2013). Participants were required to have a baseline BMD T-score of $\leq-2.5$ standard deviations (SDs) at one or more skeletal sites.

Results Overall, 222 women were included. The mean (SD) age at denosumab initiation was 64.2 (8.5) years; $26.6 \%$ reported a previous osteoporotic fracture and $6.8 \%$ a previous hip fracture. Only half of those reporting a previous fracture $(49.2 \%)$ had received prior osteoporosis therapy. At baseline, mean (SD) BMD T-scores were lumbar spine $-3.2 \mathrm{SD}$ (0.6 SD), total hip $-2.3 \mathrm{SD}$ (0.8 SD), and femoral neck $-2.7 \mathrm{SD}$ (0.7 SD). After 1 year of denosumab treatment, scores increased significantly at all three sites, reaching $-2.7 \mathrm{SD}$ (0.6 SD),
\end{abstract}

Mihail Boyanov

mihailboyanov@yahoo.com

1 University Hospital Alexandrovska, 1 St Georgi Sofiiski St, 1431 Sofia, Bulgaria

2 Medical University of Sofia, University Hospital of Endocrinology, Sofia, Bulgaria

3 Amgen (Europe) GmbH, Zug, Switzerland

4 Amgen Bulgaria, Sofia, Bulgaria
$-2.1 \mathrm{SD}(0.9 \mathrm{SD})$, and $-2.4 \mathrm{SD}(0.7 \mathrm{SD})$, respectively (all $p<0.0001$ vs. baseline). No serious adverse drug reactions were identified.

Conclusion Denosumab is usually prescribed in women with PMO at high fracture risk. In the patients who were persistent with treatment at 1 year, denosumab was well tolerated and effective at increasing BMD T-scores at several skeletal sites.

\section{Key Points}

This study reviewed the medical records of women receiving denosumab for the treatment of postmenopausal osteoporosis in Bulgaria.

The characteristics of the women suggest that they were at high risk of fracture before they were prescribed denosumab.

Denosumab increased bone mineral density after 1 year of treatment, which may help to reduce the risk of fractures in this patient population.

\section{Introduction}

Osteoporosis is characterized by low bone mineral density (BMD) and microarchitectural deterioration of bone tissue, which leads to an increased risk of fracture [1]. It has been estimated that $21 \%$ of women aged 50-84 years had osteoporosis worldwide in 2010 and that approximately 22 million women in Europe were affected [2]. These findings 
are similar to those of the largest epidemiologic osteoporosis study in Bulgaria, in which $16.8 \%$ of women aged 50 years or older were found to have osteoporosis at the femoral neck (FN) [3].

Fragility fractures are common in women over the age of 50 years, and the risk of fracture correlates with increasing age and decreasing BMD T-score [4, 5]. In Bulgaria, the mean 10-year absolute fracture risk for women with osteoporosis is reported to be $13.4 \%$ for major fractures and $2.8 \%$ for hip fractures [3]. The risk of major fractures was $8.8 \%$ in patients aged 50-59 years versus $19.6 \%$ in those aged 70 years and over, while the risk of hip fractures in these age groups was 1.0 and $6.5 \%$, respectively. Over one-fifth $(23.3 \%)$ of women reported previous low-trauma fractures, including those affecting the wrist (9.1\% of low-trauma fractures), hip (1.9\%), and vertebrae (2.3\%) [3]. Indeed, Svedbom et al. estimated that in the 1.6 million women aged 50 years or older in Bulgaria, 21,476 fractures might have occurred in 2010 [6]. However, data from the UK and Australia suggest that up to one-half of all vertebral fractures may go unrecognized $[7,8]$.

It has been estimated that, in Bulgaria in 2010, 7197 quality-adjusted life-years were lost in women aged over 50 years owing to osteoporotic fractures [6] and that the cost burden of osteoporosis was $€ 42$ million [6]. Overall, hip fractures were estimated to be the most expensive type of fracture ( $€ 20$ million), with the cost per fracture in 2010 estimated at $€ 1826$ [6].

Denosumab, a fully-human monoclonal antibody ( $\operatorname{IgG} 2)$ against RANK ligand (RANKL), was approved in Europe in 2010 for the treatment of osteoporosis in patients with an increased risk of fractures $[9,10]$. Denosumab binds RANKL with high affinity and specificity, preventing activation of its cognate receptor RANK that is expressed on the surface of osteoclasts and osteoclast precursors. Inhibition of signaling through the RANK receptor prevents osteoclast maturation, activation and survival, thereby decreasing bone resorption in cortical and trabecular bone [9]. An in vivo animal study showed that inhibition of bone resorption with denosumab improved the structural strength of bone [11, 12]. Treatment with denosumab has been associated with significant reductions in fracture risk across a wide range of patient groups $[10,13,14]$. Furthermore, long-term clinical trial follow-up data from the phase III Fracture REduction Evaluation of Denosumab in Osteoporosis every 6 months (FREEDOM) extension study demonstrated that denosumab treatment for up to 10 years was associated with a persistent reduction of bone turnover, continued increases in BMD, without therapeutic plateau, and a low fracture incidence [15]. As a consequence, Bulgarian guidelines recommend denosumab [60-mg subcutaneous injection every 6 months (SC Q6M)] as a first-line therapy to reduce the risk of vertebral, nonvertebral, and hip fractures in women with postmenopausal osteoporosis (PMO) [16].

It is of great interest to examine the profiles of patients who receive denosumab in real-world clinical practice; however, currently, such data are sparse. This study describes the baseline characteristics and changes in BMD T-scores among women with PMO who have received at least two injections of denosumab in Bulgarian clinical practice. This information may help to identify women with PMO who could gain the most benefit from treatment with denosumab.

\section{Methods}

\subsection{Study Design}

This retrospective, observational, multicenter chart review planned to include 200 women from ten representative osteoporosis practices in Bulgaria. The study protocol was approved by a central regulatory agency ethics committee, in accordance with the ethical principles of the Declaration of Helsinki.

\subsection{Participants}

Women were considered eligible for inclusion if they were aged 50 years or older, had a densitometric diagnosis of PMO [BMD T-score $\leq-2.5$ standard deviations (SDs) at one or more skeletal sites], had initiated denosumab (60 mg SC Q6M) in or after October 2011, and had received a second injection before the end of the study period (August 2013). After enrollment, patients were excluded if they had not received their second injection within 7 months of the first; this time window (6 months plus 1-month grace period) was used in accordance with the coverage period of a denosumab injection, as stated in the clinical practice guidelines for osteoporosis in Bulgaria [16], and was in line with definitions of persistence that have been used in previous studies of denosumab [17, 18]. Women were not eligible if they were currently or had ever been enrolled in a clinical trial for denosumab, if they had participated in any clinical trial in the 6 months before their first denosumab injection, or if they were contraindicated for treatment with denosumab. All patients provided informed consent to allow access to their relevant medical records.

\subsection{Study Sites and Data Collection}

The study sites were selected on the basis of geographic area, availability of electronic or paper medical records, 
and the number of women with osteoporosis treated with denosumab. At each study site, the principal investigator reviewed the medical records of postmenopausal women initiating denosumab for osteoporosis in or after October 2011 who had received a second injection within 7 months of their first injection and before the end of the study period (August 2013). Up to a maximum number of eligible women per site (which was proportional to the number of women who met the inclusion criteria at each site and was specified in the study contract) were randomly selected for inclusion via a computerized system. Women enrolled into the study were followed up from the date of their first denosumab injection until the end of the study period, and the data required as per the study protocol were then transcribed onto an electronic case report form.

\subsection{Study Outcomes}

Data for all study variables were collected from the medical records made by physicians when patients visited the clinic to receive their denosumab injections. The variables included physician-related, socio-demographic, conditionrelated (including laboratory tests performed as part of routine clinical practice), and health-related characteristics. In addition, information was collected regarding denosumab prescription and administration, procedures pertaining to denosumab administration and osteoporosis, concomitant medication use, adverse events (AEs), and adverse drug reactions (ADRs) or serious ADRs.

\subsection{BMD Measurement}

Data on BMD at baseline and at 1 year after denosumab initiation (as required for local reimbursement) were collected for one or more of the following skeletal sites: lumbar spine (LS) in the posterior-anterior position; total hip (TH); and FN. To allow the mean change in a BMD T-score between baseline and 1 year to be calculated, both measurements had to have been taken at the same skeletal site and using the same dual x-ray absorptiometry (DXA) instrument. Across the study sites, the following DXA machine models were used: Lunar Prodigy, Lunar DPX-IQ (manufactured by General Electric Healthcare, Massachusetts, USA), Hologic 1500, Hologic 2000 and Hologic C (manufactured by Hologic, Massachusetts, USA). Owing to the different instruments used across the study sites, BMD T-scores were used instead of absolute BMD values. T-scores are presented as mean (SD) unless otherwise specified.

\subsection{Statistical Analyses}

The sample size of 200 women represents approximately $1 \%$ of the women with PMO in Bulgaria. Furthermore, based on the assumption that a prevalence of $1 \%$ or more of the population would have each of the patient-related characteristics, the chances of observing at least one event among a sample of 200 patients is $87 \%$. Paired $t$ tests were used to compare BMD T-scores recorded at baseline with those recorded after 1 year of treatment with denosumab. The $\chi^{2}$ test of independence was used to verify distributional independence of outcome variables and patient groupings. A significance level of 0.05 was used.

The outcome variables LS, TH, and FN BMD T-scores were analyzed according to the following patient categories: with/without previous fracture, exposed/not exposed to prior PMO therapy, and age group. Age and prior PMO therapy were analyzed according to whether patients had a previous fracture or not. Continuous variables were summarized using mean and SD or $95 \%$ confidence intervals (CIs); categorical values were summarized as the number and proportion of women in each category.

\section{Results}

\subsection{Patient Characteristics at Baseline}

A total of 228 women who met the eligibility criteria were randomly selected for this chart review from 13 sites in Bulgaria. Overall, 222 women were included in the analyses and their baseline characteristics are presented in Table 1. Of the six women excluded from the final analyses, three had exceeded the recommended interval between denosumab injections and three were excluded at the request of their physician. The mean (SD) age at initiation of denosumab was 64.2 (8.5) years. Half of all women were younger than 65 years, and $13.1 \%$ were aged 75 years or older. Approximately one-third (30.2\%) of women had comorbidities at baseline.

Approximately one-third $(31.5 \%)$ of women had received prior osteoporosis therapy at any time during their history. The majority $(56.3 \%)$ of women were not receiving dietary supplementation with calcium or vitamin $\mathrm{D}$ at baseline (Table 1), and few women were receiving calcium-only $(5.9 \%)$ vitamin D-only $(2.7 \%)$ supplements.

More than one-quarter $(26.6 \%)$ of women reported a previous fracture. Of those reporting a previous fracture, $11.9 \%$ of women reported two previous fractures. The most common fracture type was vertebral ( $71.2 \%$ of women), followed by hip $(6.8 \%)$. Hospitalization as a result of an osteoporotic fracture was reported for $15.3 \%$ of women (Table 1).

Of those who reported a previous fracture, only half (29/ $59 ; 49.2 \%$ ) had received previous osteoporosis therapy. A $\chi^{2}$ test of independence found a statistically significant 
Table 1 Characteristics of Bulgarian women with PMO at denosumab initiation
Characteristic

Study population $(n=222)$

Age at menopause, years, mean (SD) ${ }^{\mathrm{a}}$

$48.1(4.0)$

Time since PMO diagnosis, years, mean (SD)

$1.8(4.6)$

Body mass index, $n(\%)$

$\leq 25 \mathrm{~kg} / \mathrm{m}^{2}$

$122(55.0)$

$>25 \mathrm{~kg} / \mathrm{m}^{2}$

77 (34.7)

No data

$23(10.4)$

Current smoker, $n(\%)$

Yes

$24(10.8)$

No

$192(86.5)$

Secondary osteoporosis, $n(\%)$

Yes

$6(2.7)$

No

$216(97.3)$

Age at denosumab initiation, years, mean (SD)

$64.2(8.5)$

Age at denosumab initiation, $n(\%)$

$<65$ years

$110(49.5)$

65-74 years

83 (37.4)

$\geq 75$ years

$29(13.1)$

Comorbidities, $n(\%)^{\mathrm{b}}$

$67(30.2)$

Cardiovascular disease

37 (16.7)

Metabolic disorders

$15(6.8)$

Gastrointestinal disorders

$13(5.9)$

Osteoarthritis

$6(2.7)$

Respiratory disorders

Central nervous system disorders

$4(1.8)$

3 (1.4)

3 (1.4)

Rheumatoid arthritis

$110(49.5)$

$112(50.5)$

$3.2(1.9)$

Height loss, cm, mean (SD)

Family history of osteoporotic fracture, $n(\%)$

Yes

$43(19.4)$

No

179 (80.6)

Prior PMO therapy, $n(\%)$

Yes

$70(31.5)$

No

$152(68.5)$

Calcium and/or vitamin D supplementation, $n(\%)$

Both

$78(35.1)$

Calcium only

$13(5.9)$

Vitamin D only

$6(2.7)$

None

$125(56.3)$

Previous fracture, $n(\%)$

Yes

59 (26.6)

1 fracture $^{\mathrm{c}}$

$52(88.1)$

2 fractures $^{\mathrm{c}}$

7 (11.9)

Fracture site $^{c}$

Hip

Vertebral

$42(71.2)$

Other

$19(32.2)$

Previous hospitalization for osteoporotic fracture ${ }^{\mathrm{c}}$

Hospitalization
$9(15.3)$ 
Table 1 Continued

\begin{tabular}{lc}
\hline Characteristic & Study population \\
\hline Surgery & $8(13.6)$ \\
\hline$P M O$ postmenopausal osteoporosis, $S D$ standard deviation \\
${ }^{\mathrm{a}}$ Data not available for 20 patients $(n=202)$ \\
${ }^{\mathrm{b}}$ Comorbidities that were present in $\geq 1 \%$ of patients overall are presented \\
${ }^{\mathrm{c}}$ Percentages calculated from number of patients who had experienced a previous fracture
\end{tabular}

relationship between age group and previous fracture $(p<0.0001)$. Previous fractures were more prevalent in older women: $62.1 \%$ of women aged 75 years or older had experienced a previous fracture compared with $10.0 \%$ of those who were younger than 65 years (Table 2).

The indications given for prescribing denosumab are listed in Table 3. Most women (98.6\%) were prescribed denosumab because they had a BMD T-score of less than $-2.5 \mathrm{SD}$. The next most common reasons for initiating denosumab were history of osteoporotic fracture $(22.1 \%)$ and multiple risk factors for fracture (20.3\%). Some women $(5.0 \%)$ were prescribed denosumab because they were intolerant to other therapies. More than one reason could be given per patient.

\subsection{Changes in BMD T-Scores at 1 Year}

At baseline, mean (SD) BMD T-scores were $-3.2 \mathrm{SD}$ (0.6 SD) at the LS, $-2.3 \mathrm{SD}(0.8 \mathrm{SD})$ at the $\mathrm{TH}$, and $-2.7 \mathrm{SD}$ (0.7 SD) at the FN (Fig. 1). After 1 year of denosumab treatment, mean BMD T-scores had increased significantly to $-2.7 \mathrm{SD}$ (0.6 SD), $-2.1 \mathrm{SD}(0.9 \mathrm{SD})$, and $-2.4 \mathrm{SD}(0.7 \mathrm{SD})$ at the $\mathrm{LS}, \mathrm{TH}$, and $\mathrm{FN}$, respectively (Fig. 1). Increases in BMD T-scores were calculated for the women who met the criteria of having baseline and 1-year measurements performed at the same skeletal site and using the same DXA unit. Mean $(95 \% \mathrm{CI})$ increases in BMD T-score were $0.47 \mathrm{SD}(0.42-0.53 ; n=183)$ at the LS, 0.27 SD $(0.18-0.35 ; n=64)$ at the TH, and 0.33 SD $(0.26-0.39 ; n=121)$ at the FN (all $p<0.0001$, Fig. 1$)$.

\subsection{Safety}

No new fractures or serious falls were recorded in the year following initiation of denosumab. Furthermore, no AEs, serious AEs, or ADRs related to the use of denosumab were identified from the review of the medical records.

\section{Discussion}

In this retrospective, real-world chart review of women with PMO in Bulgaria receiving at least two injections of denosumab, the drug was usually prescribed to those at high fracture risk. In the patients who were persistent with treatment at 1 year, denosumab was well tolerated and was effective in increasing BMD T-scores at several skeletal sites.

The baseline characteristics of the enrolled Bulgarian women, such as age and time since menopause, were generally similar to those of patient populations in other studies [10, 19, 20]. However, baseline BMD T-scores were lower, indicating that the women were at a high risk of fracture [4, 5, 21, 22]. The low T-scores could reflect the local reimbursement criterion for denosumab in Bulgaria, which stipulates that the cost for denosumab $60 \mathrm{mg} \mathrm{SC}$ $\mathrm{Q} 6 \mathrm{M}$ is partially reimbursed for those with a BMD T-score of $-2.5 \mathrm{SD}$ or less at one or more skeletal site.

The mean changes in BMD T-scores observed in our study were consistent with response to treatment and are similar to those reported in other studies of women with
Table 2 Previous fracture by age group in Bulgarian women with postmenopausal osteoporosis treated with denosumab

\begin{tabular}{llll}
\hline Previous fracture & \multicolumn{2}{l}{ Age group $^{\mathrm{a}}$} & \\
\cline { 2 - 4 } & $<65$ years $(n=110)$ & $65-74$ years $(n=83)$ & $\geq 75$ years $(n=29)$ \\
\hline Yes $(n=59)$ & $11(10.0)$ & $30(36.1)$ & $18(62.1)$ \\
No $(n=163)$ & $99(90.0)$ & $53(63.9)$ & $11(37.9)$ \\
\hline
\end{tabular}

Data shown are number of patients $(\%)$. Percentages are calculated from the total number of patients in each age group

a $\chi^{2}$ test of independence demonstrated a significant relationship between age group and previous fracture $\left(\chi^{2}=38.1051 ; p<0.0001\right)$ 
Table 3 Indications for prescribing denosumab for Bulgarian women with PMO

\begin{tabular}{lc}
\hline Indication & $\begin{array}{l}\text { Study population } \\
(n=222)\end{array}$ \\
\hline BMD T-score $<-2.5 \mathrm{SD}$ & $219(98.6)$ \\
History of osteoporotic fracture & $49(22.1)$ \\
Multiple risk factors for fracture & $45(20.3)$ \\
Intolerant to other PMO therapies & $11(5.0)$ \\
Patient preference for convenience and & $2(0.9)$ \\
$\quad$ safety &
\end{tabular}

Categories as reported to the Bulgarian National Health Insurance Fund; more than one reason could be provided per patient. Data are shown as $n(\%)$

$B M D$ bone mineral density, $P M O$ postmenopausal osteoporosis, $S D$ standard deviation

PMO who received treatment with denosumab. The beneficial effect of denosumab on BMD was also observed in another study over longer follow-up periods, with sustained improvements in BMD at several skeletal sites: cumulative 8 -year increases of $18.4 \%$ at the LS and $8.3 \%$ at the TH were reported [23].

It has been reported for the year 2010 that only $5 \%$ of the 240,000 women in Bulgaria eligible for treatment for PMO received it [6]. A similar care gap has been reported in other countries worldwide [24-28]. In our study, only half of the women who had experienced previous fractures, and were therefore at a high risk of subsequent fracture, had received any prior treatment for PMO. This care gap may reflect Bulgarian reimbursement requirements, whereby women with PMO need to have annual DXA scans to assess their BMD. However, the availability of DXA scanning facilities is limited; there are only 34 scanners in Bulgaria, which is equivalent to approximately five scanners per million people, and most scanners are situated in large cities, which makes access difficult for less mobile women [29]. Furthermore, the cost of PMO therapy used to be reimbursed only up to $25 \%$ and women were required to cover the remaining 75\% [29]. Consequently, many women would not have had access to PMO therapy, including denosumab. In 2014, the reimbursement rate for denosumab was increased to $50 \%$ of the total cost, which may increase patient access to therapy.

The care gap is also evident in the low proportion of women in our study who were receiving calcium or vitamin D supplementation at baseline, despite local guidelines advocating the use of these agents [29]. Calcium and vitamin D supplements are cost-effective supplements to PMO therapy [30] that have been shown to reduce bone loss and the incidence of non-vertebral fractures [31]. The low level of use of these agents is of concern and may be due to patients' fears regarding the development of kidney stones or cardiovascular disease [32].

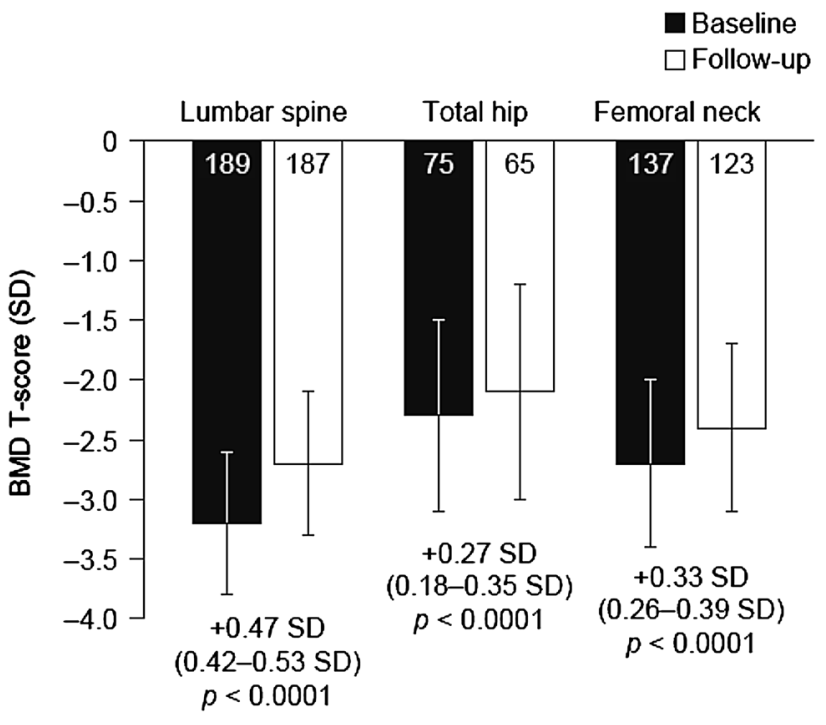

Fig.1 BMD T-scores at baseline and after 1 year of denosumab treatment in Bulgarian women with postmenopausal osteoporosis. Data are shown as mean \pm SD. Numbers within bars indicate numbers of patients. Values below bars indicate mean change in BMD T-score (95\% confidence interval). Changes in BMD were calculated only for the women who met the criteria of having baseline and 1-year measurements performed at the same skeletal site and using the same dual X-ray absorptiometry instrument $(n=183$ for lumbar spine, $n=64$ for total hip, and $n=121$ for femoral neck). Paired $t$ tests were used to compare BMD T-scores recorded at baseline with those recorded at 1-year follow-up. BMD bone mineral density, $S D$ standard deviation

Denosumab was well tolerated by the women in this chart review study. No AEs were reported during the 1-year follow-up period, although it should be noted that, owing to the retrospective nature of the study, we could not control for AEs not being recorded in patients' medical records. However, long-term data from the FREEDOM extension study have shown that the incidence of AEs did not increase over a treatment period of up to 9 years and that the incidence of fractures remained low [33, 34]. Other studies on the long-term safety of denosumab are ongoing.

There are some limitations to our study. No comparator was used; therefore, the changes in BMD T-scores between baseline and following denosumab initiation should be interpreted with care. In addition, because different DXA instruments were used at each study site, the use of absolute BMD values would not have allowed accurate grouping of data, so these data were not collected. Also, the women had received at least a second injection of denosumab within 7 months of the first injection and hence were persistent with treatment at 1 year; consequently, the results may not be generalizable to the entire population receiving denosumab for PMO, because women may not be persistent with denosumab therapy in real-world practice. However, other studies have shown that rates of persistence appear to be fairly high in routine clinical practice $[17,35]$. 
Despite the limitations, this study provides insight into the efficacy of denosumab in a persistent population of women with PMO in Bulgaria.

\section{Conclusion}

This is the first real-world chart review study of women with PMO receiving denosumab in Bulgaria. Among women receiving two denosumab injections, BMD T-scores increased significantly at several skeletal sites after 1 year. No new fractures or new safety signals were observed. Treatment with denosumab was effective in a broad population of persistent patients for 1 year. Longterm therapy with denosumab may help to reduce the incidence of fracture and the subsequent burden on women with PMO and on healthcare systems.

Acknowledgements Medical writing support was provided by Kim Allcott of Oxford PharmaGenesis, Oxford, UK. Funding for this support was provided by Amgen (Europe) $\mathrm{GmbH}$.

Authors' contributions Emi Psachoulia, Michele Intorcia, and Reneta Petkova participated in the conception and design of the overall study, and the formulation of the analysis plan. All authors were involved in the interpretation of the analyses and writing of the paper. All authors approved the final version of the paper and take responsibility for its content.

\section{Compliance with Ethical Standards}

Conflict of interest Mihail Boyanov has received speaker fees from MSD, Novartis, Roche, Amgen, Merck, and Aventis; honoraria for advisory board meetings from Amgen and MSD; and clinical trial funding from Amgen, Wyeth (Pfizer), and NPS Pharma. Alexander Shinkov has received fees as a speaker and consultant from Amgen, MSD, Servier, AstraZeneca, Hoffman-La Roche, Berlin-Chemie, Merck Serono, Abbott, Eli Lilly, and Teva. Michele Intorcia and Reneta Petkova are employees and shareholders of Amgen. Emi Psachoulia was an employee and a shareholder of Amgen at the time the study was conducted and when the manuscript was being prepared.

Ethical approval The study protocol was approved by a central regulatory agency ethics committee, in accordance with the ethical principles of the Declaration of Helsinki.

Open Access This article is distributed under the terms of the Creative Commons Attribution-NonCommercial 4.0 International License (http://creativecommons.org/licenses/by-nc/4.0/), which permits any noncommercial use, distribution, and reproduction in any medium, provided you give appropriate credit to the original author(s) and the source, provide a link to the Creative Commons license, and indicate if changes were made.

\section{References}

1. National Institutes of Health. Osteoporosis Overview. In: Osteoporosis and Related Bone Diseases National Resource
Center. 2012. http://www.niams.nih.gov/Health_Info/Bone/ Osteoporosis/overview.pdf. Accessed April 2015.

2. Hernlund E, Svedbom A, Ivergard M, et al. Osteoporosis in the European Union: medical management, epidemiology and economic burden. A report prepared in collaboration with the International Osteoporosis Foundation (IOF) and the European Federation of Pharmaceutical Industry Associations (EFPIA). Arch Osteoporos. 2013;8(1-2):136.

3. Borissova AM, Rashkov R, Boyanov M, et al. Femoral neck bone mineral density and 10-year absolute fracture risk in a national representative sample of Bulgarian women aged 50 years and older. Arch Osteoporos. 2011;6(1-2):189-95.

4. Kanis JA, Johnell O, Oden A, et al. Ten year probabilities of osteoporotic fractures according to BMD and diagnostic thresholds. Osteoporos Int. 2001;12(12):989-95.

5. Lips P. Epidemiology and predictors of fractures associated with osteoporosis. Am J Med. 1997;103(2a):3S-8S.

6. Svedbom A, Hernlund E, Ivergard M, et al. Osteoporosis in the European Union: a compendium of country-specific reports. Arch Osteoporos. 2013;8(1-2):137.

7. Gallacher SJ, Gallagher AP, McQuillian C, et al. The prevalence of vertebral fracture amongst patients presenting with non-vertebral fractures. Osteoporos Int. 2007;18(2):185-92.

8. Teede HJ, Renouf DA, Jayasuriya IA, et al. STOP fracture study: southern health osteoporotic fracture screening project. Int Med J. 2012;42(5):e74-9.

9. Amgen. Prolia ${ }^{\circledR}$ summary of product characteristics. 2014. http:// www.ema.europa.eu/docs/en_GB/document_library/EPAR_Product_Information/human/001120/WC500093526.pdf. Accessed April 2015.

10. Cummings SR, San Martin J, McClung MR, et al. Denosumab for prevention of fractures in postmenopausal women with osteoporosis. N Engl J Med. 2009;361(8):756-65.

11. Kostenuik PJ, Smith SY, Jolette J, et al. Decreased bone remodeling and porosity are associated with improved bone strength in ovariectomized cynomolgus monkeys treated with denosumab, a fully human RANKL antibody. Bone. 2011;49(2):151-61.

12. Ominsky MS, Stouch B, Schroeder J, et al. Denosumab, a fully human RANKL antibody, reduced bone turnover markers and increased trabecular and cortical bone mass, density, and strength in ovariectomized cynomolgus monkeys. Bone. 2011;49(2):162-73.

13. McClung MR, Boonen S, Torring O, et al. Effect of denosumab treatment on the risk of fractures in subgroups of women with postmenopausal osteoporosis. J Bone Miner Res Off J Am Soc Bone Miner Res. 2012;27(1):211-8.

14. Boonen S, Adachi JD, Man Z, et al. Treatment with denosumab reduces the incidence of new vertebral and hip fractures in postmenopausal women at high risk. J Clin Endocrinol Metabol. 2011;96(6):1727-36.

15. Bone HG, Brandi ML, Brown JP, et al. Ten years of denosumab treatment in postmenopausal women with osteoporosis: results from the FREEDOM extension trial American Society for Bone and Mineral Research 2015 Annual Meeting, 9-12 October, Seattle, USA 2015.

16. Borissova AM, Zacharieva S, Boyanov M, et al. Ministry of health: recommendations for good clinical practice in osteoporosis. In: Bulgarian Societies of Endocrinology and Rheumatology. 2013. http://www.iofbonehealth.org/sites/default/files/ PDFs/National\%20Guidelines/Guideline_Osteoporosis_ Bulgaria_2013_abstract.pdf. Accessed Oct 2015.

17. Silverman SL, Siris E, Kendler DL, et al. Persistence at 12 months with denosumab in postmenopausal women with osteoporosis: interim results from a prospective observational study. Osteoporos Int. 2014;26(1):361-72. 
18. Siris ES, Selby PL, Saag KG, et al. Impact of osteoporosis treatment adherence on fracture rates in North America and Europe. Am J Med. 2009;122(2 Suppl):S3-13.

19. Brown JP, Prince RL, Deal C, et al. Comparison of the effect of denosumab and alendronate on BMD and biochemical markers of bone turnover in postmenopausal women with low bone mass: a randomized, blinded, phase 3 trial. J Bone Miner Res Off J Am Soc Bone Miner Res. 2009;24(1):153-61.

20. McClung MR, Lewiecki EM, Cohen SB, et al. Denosumab in postmenopausal women with low bone mineral density. N Engl J Med. 2006;354(8):821-31.

21. Kanis JA, Johnell O, De Laet C, et al. A meta-analysis of previous fracture and subsequent fracture risk. Bone. 2004;35(2):375-82.

22. Kanis JA, McCloskey EV, Johansson H, et al. European guidance for the diagnosis and management of osteoporosis in postmenopausal women. Osteoporos Int. 2013;24(1):23-57.

23. Papapoulos S, Lippuner K, Roux C, et al. Eight years of denosumab treatment in postmenopausal women with osteoporosis: results from the first five years of the FREEDOM extension. Osteoporos Int. 2015;24(Suppl 2):Abstr OC21.

24. Jennings LA, Auerbach AD, Maselli J, et al. Missed opportunities for osteoporosis treatment in patients hospitalized for hip fracture. J Am Geriatr Soc. 2010;58(4):650-7.

25. Kung AW, Fan T, Xu L, et al. Factors influencing diagnosis and treatment of osteoporosis after a fragility fracture among postmenopausal women in Asian countries: a retrospective study. BMC Womens Health. 2013;13:7.

26. McGowan B, Bennett K, Casey MC, et al. Comparison of prescribing and adherence patterns of anti-osteoporotic medications post-admission for fragility type fracture in an urban teaching hospital and a rural teaching hospital in Ireland between 2005 and 2008. Irish J Med Sci. 2013;182(4):601-8.

27. Teede HJ, Jayasuriya IA, Gilfillan CP. Fracture prevention strategies in patients presenting to Australian hospitals with minimal-trauma fractures: a major treatment gap. Int Med J. 2007;37(10):674-9.

28. Munson JC, Bynum JW, Bell J, et al. Patterns of prescription drug use before and after fragility fracture. JAMA Int Med. 2016;176(10):1531-8.

29. Borissova AM, Kovatcheva R, Boyanov M, et al. Eastern European and central Asian regional audit-epidemiology costs and burden of osteoporosis in 2010. http://www.iofbonehealth.org/ sites/default/files/PDFs/Audit\%20Eastern\%20Europe_Central\% 20Asia/Russian_Audit-Bulgaria.pdf. Accessed Oct 2015.

30. Hiligsmann M, Ben Sedrine W, Bruyere O, et al. Cost-effectiveness of vitamin D and calcium supplementation in the treatment of elderly women and men with osteoporosis. Eur J Public Health. 2015;25:20-5.

31. Dawson-Hughes B, Harris SS, Krall EA, et al. Effect of calcium and vitamin D supplementation on bone density in men and women 65 years of age or older. $N$ Engl $J$ Med. 1997;337(10):670-6.

32. Manson JE, Bassuk SS. Calcium supplements: do they help or harm? Menopause. 2014;21(1):106-8.

33. Papapoulos S, Roux C, Bone HG, et al. Denosumab treatment in postmenopausal women with osteoporosis for up to 9 years: results through year 6 of the FREEDOM extension. Ann Rhem Dis. 2015;74(Suppl 2).

34. Papapoulos S, Roux C, Bone HG, et al. Denosumab treatment in postmenopausal women with osteoporosis for up to 9 years: results through year 6 of the FREEDOM extension. Osteoporos Int. 2015;26 (Suppl 1):Abstr OC4.

35. Hadji P, Papaioannou N, Gielen E, et al. Persistence, adherence, and medication-taking behavior in women with postmenopausal osteoporosis receiving denosumab in routine practice in Germany, Austria, Greece, and Belgium: 12-month results from a European non-interventional study. Osteoporos Int. 2015;26:2479-89. 